\title{
The prevalence of clostridium difficile infection in a cohort of Sri Lankan patients with acute severe ulcerative colitis
}

\begin{abstract}
Introduction: Global studies report that 5\%-19\% of patients with acute severe ulcerative colitis (ASUC) are positive for Clostridium difficile. Data from Sri Lanka, as well as from other countries in South Asia are lacking. Guidelines recommend all patients with ASUC be tested for Clostridium difficile in stools. The implications of this on developing countries such as ours are a higher cost to patients. Therefore in this study we sort to find out the prevalence of Clostridium difficile infection in patients with ASUC presenting to a teaching hospital over an eighteen month period.
\end{abstract}

Method: All ulcerative colitis (histologically proven) patients admitted with ASUC from July 2011 to December 2012 were included. ASUC was diagnosed according to True love and Witt's criteria. A Clostridium difficile infection was diagnosed based on the ELISA test to detect Clostridium difficile toxins, done on a single sample of stools within 24 hours of admission.

Results and discussion: 32 patients; with ASUC were included. Six (18.75\%) tested positive for Clostridium difficile. The mean age was 28 and 48 years in the positive and negative group respectively. Of the positive patients three were previously on Aminosalicylates and three were treatment naive. Apart from one patient with a history of recent antibiotic use, none of the other patients gave a history of antibiotic, PPI use or hospital admission within 3 months. The White blood cell count, ESR or stools full report were unable to predict the presence of Clostridium difficile infection. Apart from one patient with pseudomembranes on endoscopy, none of the other toxin positive patient's endoscopy or histology was suggestive of Clostridium difficile infection.

Conclusion: This study shows a high prevalence of Clostridium difficile infection among Sri Lankan patients presenting with ASUC, which is in keeping with studies reported previously. Although local or regional guidelines are lacking, this study also reconfirms the necessity to test for Clostridium difficile in ASUC. As suggested by previous authors the risk factors associated with the development of Clostridium difficile infections in ASUC seems different to the general population.

Keywords: acute severe ulcerative colitis, clostridium difficile infection
Volume I Issue 2 - 2014

\author{
Fernandopulle ANR, Mayadunne PB, \\ Navarathne NMM \\ Gastroenterology and Hepatology Unit, National hospital of \\ Sri Lanka
}

Correspondence: Fernandopulle ANR, Consultant Gastroenterologist, National Hospital of Sri Lanka, EW Perera Mawatha, Colombo, Sri Lanka, Tel 94-77-3-422454, Email anrfernandopulle@yahoo.com

Received: May II, 20I4 | Published: June 27, 2014
Abbreviations: ASUC, acute severe ulcerative colitis; WBC, white blood cells; RBC, red blood cells; IBD, inflammatory bowel disease; ECCO, european crohn's and colitis organization; CRP, c reactive protein; PPI, proton pump inhibitors; ELISA, enzyme immune sorbent assay; ESR, erythrocyte sedimentation rate; SFR, stools full report

\section{Introduction}

Ulcerative colitis is a chronic inflammatory bowel disease with periods of remission and exacerbation and varying prognosis depending on the severity of the disease. Clostridium difficile associated diarrhea has become a major health hazard throughout the world and is responsible for many hospital related morbidly and mortality. ${ }^{1}$ Recognized risk factors for infection include advanced age ( $>65$ years old), recent $(<2$ months) broad-spectrum antibiotic therapy, long hospital stay, and presence of multiple other co morbidities. ${ }^{2-4}$ Clostridium difficile infection is also becoming more severe and resistant to antibiotics, conferring a mortality rate of $1-2 \%$ in such cases. 4

In the literature there are several reports on the impact of Clostridium difficile on ulcerative colitis. ${ }^{5,6}$ Unfortunately all these reports are from the east and west, and there is a scarcity of such reports from the South Asian region. ${ }^{7}$ From the available literature the prevalence of Clostridium difficile in acute severe ulcerative colitis is between 5-19\% in various regions. ${ }^{8}{ }^{89}$ Guidelines published by the European Crohn's and Colitis Organization (ECCO) propose that Clostridium difficile should be examined in all patients presenting with an acute episode of Ulcerative colitis. ${ }^{10}$ Unfortunately we do not have local or regional guidelines on the management of inflammatory bowel disease in this part of the world and are dependent on the above guidelines. While we accept and use these guidelines to manage are patients, it has to be understood that these recommendations are based on the available studies from the west, and there may be certain unique differences in the clinical profiles of our patients. ${ }^{11}$ Therefore, the impact of such recommendation on developing countries such as those in South Asia is twofold. If the prevalence of Clostridium difficile in ulcerative colitis is actually as high as in the western studies, early detection and appropriate treatment would obviously reduce longer hospitalization and additional costs. On the contrary if the prevalence is low, the cost of performing expensive tests to detect Clostridium difficile infection would be counterproductive and alternative methods to identify high 
risk patients need to be found out. This would also mean that we need to tailor some the recommendation from international guidelines to suit our patients, so that an efficient and cost effective management could be provided. There is only a single report from India, on the role of infectious agents during exacerbation in ulcerative colitis, ${ }^{7}$ and to date no reports on the prevalence of Clostridium difficile in acute severe ulcerative colitis from this region. Therefore, in this study we looked at the prevalence of ulcerative colitis in a cohort of Sri Lankan patient's presenting with acute severe ulcerative colitis.

\section{Method}

We prospectively collected data from all the patients admitted with acute severe ulcerative colitis (ASUC) to the National hospital of Sri Lanka during an eighteen month period from July 2011 to December 2012. All patients had histological confirmation of disease either before or after admission, in case it was a new presentation. Severity was defined using the True love and Witt's criteria, Bloody stools of more than 6 times a day with either, pulse rate $>90$ beats per min, erythrocyte sedimentation rate (ESR) $>30 \mathrm{~mm}$, temperature $>37.7$ Celsius or hemoglobin $<10.5 \mathrm{~g} / \mathrm{dl}$. Stools were collected from all patients on admission before any treatment was initiated in hospital. Stools samples were sent to the laboratory in a plastic container according to the appropriate guidelines for transporting stools for Clostridium difficile assay. Clostridium difficile Toxins were analyzed using the Enzyme immune sorbent assay (ELISA) for toxins A and B at the Medical Research Institute of Sri Lanka. All patients had an unprepared sigmoidoscopy examination within 24-48hrs of admission. The typical text book appearance of pseudo membranes in the colon was used as the endoscopic appearance of Clostridium difficile. Non specific mucosal changes such as edema, granularity, vascularity, bleeding and ulceration which were similar to ulcerative colitis were not taken as suggestive endoscopic changes. Routine blood investigations were sent in all patients at admission. A high white blood cell count was considered as above $11,000 / \mathrm{cm}^{3}$. Stools full report was used to look for red blood cells (RBC), white blood cells (WBC) and any other pathogens. If any RBC's were detected with WBC the test was considered positive. Above $30 \mathrm{~mm}$ was taken as a high ESR. C reactive protein (CRP) could not be routinely performed in all patients due to financial constraints. We collected data regarding medications for ulcerative colitis and if the patient was on proton pump inhibitors (PPI) or antibiotics during the preceding four weeks and were hospitalized within the preceding three months, as these were considered risk factors for Clostridium difficile infection in previous studies. The data was collected on direct questioning of the patient during the admission after informed consent was obtained to participate in this study. Approval for the study was obtained by the institutional ethical review committee of the National hospital of Sri Lanka.

\section{Results}

There were thirty two admissions of acute severe ulcerative colitis during the study period. The mean age of the study group was 48 years (21-70yrs). Seven (18.75\%) out of 32 patients tested positive for Clostridium difficile toxin A. For purpose of analysis Clostridium difficile positive and negative patients were considered as Group A and $\mathrm{B}$ respectively. The mean age was 28 and 48 years in group A and group $\mathrm{B}$ respectively. The presence of fever, medications patients were on admission, hospitalization within the past 3 months, white blood cell count (WBC), erythrocyte sedimentation rate (ESR) and stools full report (SFR) report were analyzed between the two groups and the results are summarized in Table 1. In group A, three patients were previously on Aminosalicylates and three were treatment naive. The three patients on treatment were already diagnosed with ulcerative colitis; the latter three were new diagnoses. None of the group A patients were on other stronger immune suppressants like Azathioprine, Methotrexate or biologics. In group a, 1/6 gave a history of recent antibiotic use but none had taken proton pump inhibitor or been admitted to hospital within 3 months. The presence of fever, WBC, ESR or stools full report were not significantly different between the two groups (Table 2). Apart from one patient where the endoscopy was suggestive of Clostridium difficile infection none of the other group A patients had suggestive endoscopy or histology findings.

Table I Summary of the Clostridium difficile toxin positive and negative groups

\begin{tabular}{|c|c|c|c|c|}
\hline \multirow[b]{2}{*}{ New diagnosis } & \multicolumn{2}{|c|}{ Group A (Toxin Positive) } & \multicolumn{2}{|c|}{ Group B (Toxin Negative) } \\
\hline & $3 / 6$ & & $4 / 26$ & \\
\hline Sulphasalazine use & $3 / 6$ & & $22 / 26$ & \\
\hline Azathioprine use & $0 / 6$ & & $5 / 26$ & \\
\hline Fever & None & & None & \\
\hline ESR & High (4/6) & Low $(2 / 6)$ & $\operatorname{High}(10 / 26)$ & Low $(15 / 26)$ \\
\hline WBC count & High (4/6) & Low $(2 / 6)$ & High (13/26) & Low $(12 / 26)$ \\
\hline Stools full report & Positive (5/6) & Negative (I/6) & Positive (20/26) & Negative $(5 / 26)$ \\
\hline Endoscopy & Suggestive (1/6) & Not suggestive $(5 / 6)$ & \multirow[b]{2}{*}{$s$} & \\
\hline Histology & Suggestive $(0 / 6)$ & Not suggestive $(6 / 6)$ & & \\
\hline
\end{tabular}

Table 2 Comparison of known risk factors for Clostridium difficile infection and suggestive clinical parameters to suspect Clostridium difficile infection

\begin{tabular}{llll}
\hline & Group A (Toxin Positive) & Group B (Toxin Negative) & P Value \\
\hline Prior antibiotics & $\mathrm{I}$ & 0 & \\
Recent hospitalisation & 0 & 0 & \\
ESR above 30mm & $4 / 6$ & $10 / 26$ & 0.419 \\
Positive stools full report & $5 / 6$ & $20 / 26$ & 0.628 \\
High WBC count & $4 / 6$ & $13 / 26$ & 0.810 \\
Fever & $0 / 6$ & $0 / 26$ & \\
\hline
\end{tabular}




\section{Discussion}

For decades, Clostridium difficile has been recognized as a cause of diarrhea and most clinicians are familiar with the settings in which Clostridium difficile infection is considered high risk. Although antibiotics have long been considered the "culprit" behind most Clostridium difficile infection, recently there seems to be substantial evidence that proton pump inhibitors may be incriminated with similar effect. ${ }^{12,13}$ However there also seems to be an additional subset of inflammatory bowel disease (IBD) patients who are at a higher risk of acquiring Clostridium difficile disease. The prevalence rates of Clostridium difficile infection in severe ulcerative colitis have been as high as $19 \%$ in some studies with a range of 5-19\%. ${ }^{8,9}$ Clostridium difficile infection is also thought to be associated with worse prognosis when present during such exacerbation of ulcerative colitis. ${ }^{14}$ Due to these high prevalence rates of Clostridium difficile infection in acute severe ulcerative colitis and the poorer prognosis, it is recommended in most Ulcerative colitis management guidelines that all patients with severe exacerbations of ulcerative colitis be investigated for Clostridium difficile infection. The implications of such a recommendation needs to be thought of especially in low income countries such as ours. Although the high prevalence rates of $5-19 \%$ are reported in western studies. ${ }^{8,9}$ there is a scarcity of data in countries in South Asia, where patient management cost weighs high in decision making.

In our study population which included thirty two patients with acute severe Ulcerative colitis, (6/32) $18.75 \%$ were found to be positive for Clostridium difficile toxins. It is thought that inflammatory bowel disease patients may be at a higher risk of Clostridium difficile infection even without the known risk factors of previous hospital admission, antibiotic or PPI use. One of the reasons postulated has been the immune compromised state of the IBD patients, particularly those who are on immune suppressant medications..$^{15}$ Interestingly none of our patients were on strong immune suppressants other than aminosaliasylates. Antibiotic are known and reported to be over used and may be even misused in most South Asian countries. Our patients denied using any antibiotics prior to admission. It is possible that patients conceal their previous medications in order to get new medication. Therefore given the liberal use of antibiotics in our country, we feel it may still have some role in explaining such high prevalence figures in our study.

In previous literature up to $76 \%$ of Clostridium difficile infections in inflammatory bowel disease patients were detected in an outpatient setting. ${ }^{16}$ This lends support to our findings, where none of the patients had been recently admitted to hospital either. It seems a large proportion of inflammatory bowel disease patients acquire infection before hospitalization, or that many of these infections result in hospitalization. Thus, at least in ulcerative colitis Clostridium difficile infection appears to be more often a cause of hospitalization rather than the result of it. This again seems to be unique to ulcerative colitis patients.

Pseudo membranes on endoscopy have long been synonymous with Clostridium difficile infection although other causes have been implicated later. ${ }^{17}$ Interestingly, Clostridium difficile infection in ulcerative colitis does not seem to uniformly produce pseudo membranous changes when the colon is examined endoscopically, at least in the acute setting. In the study by Issa et al. ${ }^{16}$ none of the sixteen ulcerative colitis patients with positive Clostridium difficile stool assays demonstrated pseudo membranes either macroscopically or histologically. Similarly, in our study apart from one patient (1/6, $16.6 \%$ ) we did not find suggestive endoscopy findings or histology reports in any of our toxin positive patients. This again has cost implications for developing countries like ours where endoscopy and histology may not be considered "expensive" investigations, owing to the fact that they are performed free of charge in state hospitals. But a Clostridium difficile toxin assay may cost the patient additional money in most hospitals. Thus, as Gastroenterologists we have to always bear in mind that the typical endoscopic features and histological confirmation are lacking in this sub set of patients.

At least in resource poor settings it is important that clinicians use a pragmatic but evidence based approach when managing patients with ASUC. Historically some clinicians have suspected Clostridium difficile infection in ASUC if the CRP level is extremely high, the WBC count is considerably elevated or the patients have high fever spikes. If accurate, this would obviously be a simple clinical tool to risk stratify patients with ASUC and decide if investigation of Clostridium difficile is needed and thereafter if empirical treatment should be started. Unfortunately in our study we could not find such a statistically significant relationship between the above three variables and Clostridium difficile toxin positivity. The limitation of this finding is that the number of patients with positive Clostridium difficile toxins in our study was small for a reliable conclusion to be made. But as this has not been studied previously it is worth highlighting for a future larger study.

\section{Conclusion}

As shown in this study, the prevalence of Clostridium difficile infection in ASUC patients in this region is high (18.75\%), and although expensive, it is important that we test for Clostridium difficile in ASUC.

In countries which lack its own guidelines to manage ulcerative colitis and therefore follow international guidelines, it is important that such regional epidemiological data are available, as it has implication on its health budget. It is also important that we have our own studies to indentify the clinical profiles of our patients as they may sometimes be unique and different to what is seen in the west. Unlike in the past, there is an increasing prevalence of IBD in developing countries like Sri Lanka and in Asia as a whole. Therefore it is appropriate that we have our own epidemiological data to guide clinicians in their decision making. And as shown in this study if our own studies correspond with international findings, we can follow these recommendations and where they differ, we need to tailor such recommendation to suit our patients. While this will bring uniformity in management of ulcerative colitis, it will also make management efficient and cost effective.

\section{Conflicts of Interest}

Fernandopulle ANR conceived and designed this study, collected data, performed the statistical analysis and drafted the manuscript. Mayadunne PB helped collect data. Navarathne NMM helped in its design and coordination and helped to draft the manuscript.

\section{Acknowledgements}

None.

\section{Funding}

None. 


\section{References}

1. Dallal RM, Harbrecht BG, Boujoukas AJ, et al. Fulminant Clostridium difficile: an underappreciated and increasing cause of death and complications. Ann Surg. 2002;235(3):363-372.

2. McDonald CL, Owings M, Jernigan DB. Clostridium difficile infection in patients discharged from US short-stay hospitals, 1996-2003. Emerg Infect Dis. 2006;1(3)2:409-415.

3. Bartlett JG. Antibiotic-associated diarrhea. N Engl J Med. 2002;346(5) 334-339.

4. Barbut F, Petit JC. Epidemiology of Clostridium difficile-associated infections. Clin Microbiol Infect. 2001;7(8):405-410.

5. Rodemann JF, Dubberke ER, Reske KA, et al. Stone CD Incidence of Clostridium difficile infection in inflammatory bowel disease. Clin Gastroenterol Hepatol. 2007;5(3): 339-344.

6. Tremaine WJ. Inflammatory bowel disease and Clostridium difficileassociated diarrhea: a growing problem. Clin Gastroenterol Hepatol. 2007;5(3):310-311

7. Kochhar R, Ayyagari A, Goenka MK, et al. Role of infectious agents in exacerbations of ulcerative colitis in India. A study of Clostridium difficile. J Clin Gastroenterol. 1993;16(1):26-30.

8. Meyer AM, Ramzan NN, Loftus EV, et al. The diagnostic yield of stool pathogen studies during relapses of inflammatory bowel disease. J Clin Gastroenterol. 2004;38(9):772-775.

9. Mylonaki M, Langmead L, Pantes A, et al. Enteric infection in relapse of inflammatory bowel disease: importance of microbiological examination of stool. Euro J Gastroenterol Hepatol. 2004;16(8):775-778.
10. Dignass A, Eliakim R, Magro F, et al. Second European evidencebased consensus on the diagnosis and management of ulcerative colitis: definitions and diagnosis. J Crohns Colitis. 2012;6(10):965-990.

11. Senanayake SM, Fernandopulle AN, Niriella MA, et al. The long-term outcomes of a cohort of Sri Lankan patients with ulcerative colitis: a retrospective study at two national referral centers and review of literature. Clin Exp Gastroenterol. 2013;6:195-200.

12. Janarthanan S, Ditah I, Adler DG, et al. Clostridium difficile-associated diarrhea and proton pump inhibitor therapy: a meta-analysis. Am J Gastroenterol. 2012;107(7):1001-1010.

13. Pohl JF. Clostridium difficile infection and proton pump inhibitors. Curr Opin Pediatr. 2012;24(5):627-631.

14. Adams SD, Mercer DW. Fulminant Clostridium difficile colitis. Curr Opin Crit Care. 2007;13(4): 450-455.

15. Bignardi GE. Risk factors for Clostridium difficile infection. J Hosp Infect. 1998;40(1):1-15.

16. Issa $\mathrm{M}$, Skaros $\mathrm{S}$, Beaulieu DB. Increasing impact of Clostridium difficile on inflammatory bowel disease: a tertiary referral center experience (abstract W1168). Gastroenterology. 2006;130:A-649.

17. Seppala K, Hjelt L, Sipponen P. Colonoscopy in the diagnosis of antibiotic-associated colitis. A prospective study. Scand J Gastroenterol. 1981;16(4): 465-468. 